\title{
Caracterização reológica de pasta cimentícia: associação de técnicas complementares
}

\author{
Rheological characterization of cement paste: association \\ of complementary techniques
}

\author{
Andrea Murillo Betioli \\ Vanderley Moacyr John \\ Philippe Jean Paul Gleize \\ Rafael Giuliano Pileggi
}

Andrea Murillo Betioli Departamento de Engenharia de Construção Civil, Escola Politécnica Universidade de São Paulo Avenida Professor Almeida Prado, Trav. 2, n. 83, Cidade Universitária Caixa-Postal: 476 São Paulo - SP - Brasil CEP 05508-900

Tel.: (11) 3091-5382 E-mail: andreabetioli@gmail.com

Vanderley Moacyr John Departamento de Engenharia de Construção Civil, Escola Politécnica Universidade de São Paulo Tel.: (11) 3091-5794 E-mail:

vanderley.john@poli.usp.br

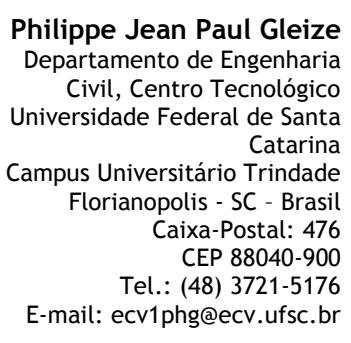

Rafael Giuliano Pileggi Departamento de Engenharia de Construção Civil, Escola Politécnica

Universidade de São Paulo Tel.: (11) 3091-5248
E-mail:

rafael.pileggi@poli.usp.br

Recebido em 14/07/09 Aceito em 16/11/09

\section{Resumo

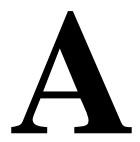

adaptabilidade a diferentes tipos de materiais (concretos, argamassas, fibrocimento, etc.) e aplicações (injeção, bombeamento, spray, extrusão, etc.) é essencial para utilização de materiais à base de cimento. Cada material ou sistema de aplicação demanda um conjunto de características específicas da pasta fluida. Portanto, um ensaio adequado para avaliar o comportamento reológico destes materiais deve identificar todas as propriedades requeridas para cada situação. A maior dificuldade está na obtenção de uma única técnica capaz de simular todas as situações simultaneamente. Como alternativa, este trabalho propôs avaliar o comportamento reológico de uma pasta de cimento por uma associação de técnicas reológicas, tais como: reometrias rotacionais tradicionais de fluxo e oscilação e, um método menos explorado, o squeeze-flow. Os resultados mostraram que uma única técnica não é capaz de determinar as propriedades reológicas fundamentais nem de simular todas as solicitações que os materiais podem sofrer durante sua mistura, transporte e aplicação. Técnicas complementares foram necessárias, permitindo assim identificar o comportamento da pasta de cimento sob diferentes solicitações.

Palavras-chave: Pasta de cimento. Técnicas de caraterização reológica. Reometrias de fluxo e oscilatória. Reometria squeeze-flow.

\section{Abstract}

The adaptability to different classes of material (concrete, mortars, fiber-cement, etc.) and methods of application (injection, pumping, spraying, casting, extrusion, etc.) is an essential feature of cement pastes. Each material or application system requires a set of characteristics of the fluid pastes. Therefore, an adequate rheological test to characterize rheological behavior should identify all the required properties for each situation. The main problem associated with this approach is the lack of a single technique able to perform a thorough characterization. As an alternative, this work proposes to evaluate the behavior of a cement paste by associating traditional flow and oscillatory rheometry, with the less explored squeeze-flow testing method. The results showed that a single technique cannot evaluate the fundamental properties nor simulate the mixing, transport and application methods. Complementary techniques were necessary to identify the rheological behavior under different requirements.

Keywords: Cement paste. Rheological characterization. Flow and oscillatory rheometry. Squeeze-flow rheometry. 


\section{Introdução}

Concretos (convencionais, autoadensáveis, de alto desempenho, bombeados, microconcretos, concretos com fibras), argamassas (revestimento, assentamento, bombeáveis, projetadas) e fibrocimento podem ser descritos resumidamente como materiais multifásicos compostos de uma fração grossa inerte (areia e brita), uma fração de finos reativa (cimento, cal, microssílica, etc.), fibras (sintéticas, naturais) e ar incorporado.

Em condições de cisalhamento no estado fresco (período de indução), esses materiais podem ser misturados e aplicados de diferentes maneiras, conforme a necessidade, como é o caso, por exemplo, de aplicações por injeção, spray, extrusão e bombeamento. Essa variedade de materiais e aplicações requer diferentes características reológicas. Além dessa variedade, a reatividade do cimento torna a reologia desses materiais ainda mais complexa.

As alterações de consistência desses materiais observadas em função do tempo são atribuídas às reações químicas de hidratação do cimento dissolução/precipitação (TAYLOR, 1990) - e aos fenômenos físicos, como aglomeração das partículas em meio aquoso, resultado das forças eletrostáticas e forças de van der Waals; alta força iônica do meio aquoso e a formação de produtos hidratados entre as partículas de cimento (YANG; JENNINGS, 1992; JIANG et al., 1995; NACHBAUR et al., 1998, 2001; BETIOLI et al., 2008).

Corriqueiramente, tais alterações são avaliadas por ensaios como cone de Marsh e mini-slump para pastas; mesa de consistência, dropping-ball, cone de penetração, Vane Test, para argamassas; e slump-test para concretos, entre outros. No entanto, esses ensaios, denominados de ensaios de monoponto, estão relacionados a uma única taxa de cisalhamento, que permite a mensuração indireta de um único parâmetro reológico fundamental, a tensão de escoamento ou a viscosidade, e, por isso, não são eficientes para avaliar o estado estático ou sob fluxo (HOPPE et al., 2006).

Com o surgimento e a evolução dos reômetros, a determinação dos parâmetros reológicos fundamentais tornou-se possível em diferentes taxas de cisalhamento, obtendo-se, dessa forma, o perfil reológico do material. Outra vantagem é a possibilidade de simular os processos relacionados à mistura, transporte e aplicação variando-se a taxa de cisalhamento por meio de ensaios de fluxo. Ainda, dependendo do tipo de reômetro, é possível avaliar a consolidação desses materiais, isto é, avaliar as alterações viscoelásticas associadas à pega do cimento, sendo o ensaio oscilatório o mais adequado para essa situação. Ambos os métodos são classificados como reometria rotacional e com geometria constante durante a medida.

Em outros processos, como extrusão, formação por compressão e aplicações submetidas ao cisalhamento e à elongação, como é o caso do cisalhamento sofrido pela pasta entre os agregados de argamassas e concretos, a restrição geométrica resulta em diferentes propriedades, como o atrito, especialmente para suspensões concentradas, como é o caso dos materiais à base de cimento. Para simular esse tipo de esforço, o ensaio de squeezeflow seria o mais apropriado (MIN et al., 1994; ÖZKAN et al., 1999; MEETEN, 2000; ENGMANN et al., 2005; PHAN et al., 2006; STEFFE; 1996). Além disso, esse ensaio evita problemas como deslizamento, rompimento de materiais plásticos e a dificuldade em avaliar materiais muito viscosos ou com adição de fibras.

Embora possa apresentar a mesma geometria (placa/placa), como é o caso deste trabalho (Figura 1), cada método (fluxo, oscilatório e squeeze-flow) fornece uma solicitação reológica com diferente magnitude de deformação: alta para ensaio de fluxo e squeeze-flow, e baixa para oscilatório, além da diferença nas restrições geométricas. A Tabela 1 mostra os equipamentos e os parâmetros determinados em cada método.

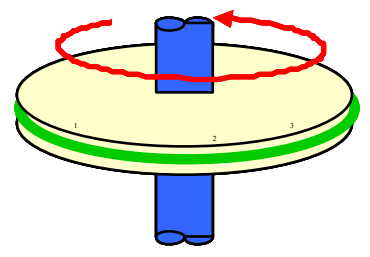

(a) Ensaio de fluxo

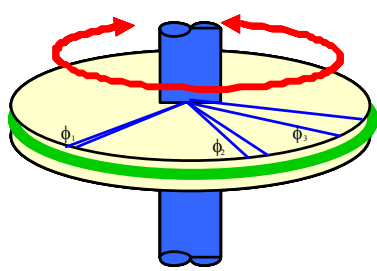

(b) Ensaio oscilatório

Obs: a mesma geometria (placa/placa) promove solicitações diferentes Figura 1 - Ilustração esquemática dos métodos reológicos

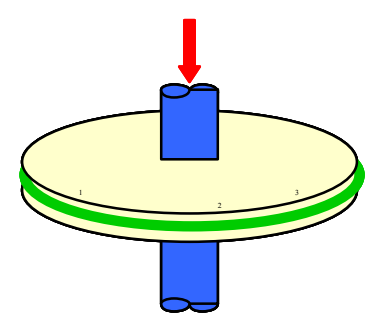

(c) Squeeze-flow 


\begin{tabular}{c|c|c}
\hline Reometria & Equipamento & Parâmetros determinados \\
\hline Fluxo & Reômetro & Perfil de fluxo, tensão de escoamento e viscosidade \\
\hline Oscilação & Reômetro & $\begin{array}{c}\text { Módulo de armazenamento }\left(\mathrm{G}^{\prime}\right), \text { módulo de perda } \\
(\mathrm{G} \text { '’) e tensão de escoamento }\end{array}$ \\
\hline Squeeze-flow & $\begin{array}{c}\text { Prensa ou } \\
\text { reômetro }\end{array}$ & $\begin{array}{c}\text { Perfil da curva carga } x \text { deslocamento, carga máxima, } \\
\text { tensão de escoamento, viscosidade elongacional }\end{array}$ \\
\hline
\end{tabular}

Tabela 1 - Descrição dos ensaios reológicos, equipamentos e parâmetros determinados

\begin{tabular}{|c|c|c|}
\hline \multirow{2}{*}{\multicolumn{2}{|c|}{ Finura - resíduo na neneira 75 um (\%) (NBR 11579·1991) }} & \\
\hline & & 3,2 \\
\hline \multicolumn{2}{|c|}{ Área específica $\left(\mathrm{m}^{2} / \mathrm{g}\right)^{*}$} & 1,22 \\
\hline \multicolumn{2}{|l|}{ Início; Fim de pega (minutos) } & $185 ; 285$ \\
\hline \multirow{3}{*}{$\begin{array}{l}\text { Resistência à compressãc } \\
7215: 1996)\end{array}$} & 3 dias & 25,8 \\
\hline & 7 dias & 31,9 \\
\hline & 28 dias & 40,0 \\
\hline
\end{tabular}

*Picnômetro de gás hélio

Tabela 2 - Características físicas do cimento Portland

* Cálculo de Bogue

\begin{tabular}{l|c}
\hline Perda ao fogo (\%) & 5,10 \\
\hline Resíduo insolúvel (\%) & 0,76 \\
\hline $\mathrm{C}_{3} \mathrm{~S}^{*}(\%)$ & 45 \\
\hline $\mathrm{C}_{2} \mathrm{~S}^{*}(\%)$ & 19 \\
\hline $\mathrm{C}_{3} \mathrm{~A}^{*}(\%)$ & 6 \\
\hline $\mathrm{C}_{4} \mathrm{AF}^{*}(\%)$ & 9 \\
\hline
\end{tabular}

Tabela 3 - Características químicas do cimento Portland

Perante o exposto, a maior dificuldade na caracterização está na obtenção de uma única técnica capaz de simular todas as situações simultaneamente e, por isso, o objetivo principal deste trabalho foi avaliar o comportamento reológico de uma pasta de cimento sob diferentes solicitações por meio de ensaios reológicos rotacionais de fluxo e oscilatório, e sob compressão (squeeze-flow).

\section{Materiais e métodos}

O cimento utilizado neste estudo foi o cimento Portland composto de calcário moído do tipo CPIIF 32, em razão de ele não possuir adições reativas, tais como pozolanas e escória de alto-forno. As características físicas e químicas encontram-se nas Tabelas 2 e 3.

A relação água/cimento foi de 0,38 , e a mistura da pasta foi realizada em um misturador IKA RW 20 DZM.n, seguindo os passos descritos a seguir: adição do cimento sobre a água de modo contínuo, com o misturador ligado na rotação de $300 \mathrm{rpm}$, durante aproximadamente $3 \mathrm{~min}$; concluída a adição, mistura por mais $2 \mathrm{~min}$, na mesma velocidade.

Os ensaios reológicos tiveram início aos $15 \mathrm{~min}$ de hidratação, contados a partir do contato do cimento com a água. As medidas reológicas foram realizadas até 60 min de hidratação, garantindo, assim, que elas fossem realizadas no período de indução (TAYLOR, 1990), comprovado pelo ensaio de calorimetria realizado em calorímetro de condução JAF (Wexham Developments) (temperatura constante de $25^{\circ} \mathrm{C}$ ), com sistema de aquisição informatizado com coleta de dados a cada 3 min.

O período de indução entre 20 min e 110 min foi calculado a partir das intersecções do trecho horizontal com duas retas: a reta traçada no período de pré-indução e a extrapolação da linha de regressão do período de aceleração (SILVA, 2001), como demonstrado na Figura 2.

\section{Ensaio de fluxo}

Este ensaio pode ser utilizado para simular misturas e aplicações, e estudar o efeito de matérias-primas, aditivos, adições, entre outros. Denominado de ensaio estático, o ensaio de fluxo mede a tensão de cisalhamento a uma dada taxa de cisalhamento (ou vice-versa). Com a variação da taxa ou da tensão de cisalhamento, a curva de escoamento pode ser traçada e, através desta ou de equações de estado reológico, as propriedades reológicas podem ser determinadas - viscosidade (resistência ao escoamento) e tensão de escoamento (tensão mínima necessária para iniciar o escoamento do material). O comportamento 
reológico pode ser apresentado por curvas de tensão $x$ taxa de cisalhamento ou viscosidade $x$ taxa de cisalhamento.

As medidas neste trabalho foram realizadas em reômetro rotacional com tensão controlada (AR 2000, TA Instruments). A geometria escolhida foi a de placas paralelas, com diâmetro de $40 \mathrm{~mm}$ e espaçadas de $1 \mathrm{~mm}$. Um adesivo texturizado foi colocado na superfície das placas para se evitar o deslizamento da amostra. Utilizou-se um dispositivo para evitar a evaporação de água durante o ensaio.

$\mathrm{O}$ ciclo de histerese foi realizado por meio de um ensaio de rampa com aceleração de 0 a $100 \mathrm{~s}^{-1} \mathrm{em}$ 2 min e, imediatamente depois, uma desaceleração até 0 , em mais 2 min (Figura 3). Realizaram-se os ciclos de cisalhamento em tempos predefinidos, 15 min e $60 \mathrm{~min}$, utilizando-se uma amostra para cada tempo. Foram realizadas duas repetições para cada amostra.
A viscosidade aparente foi determinada pela razão entre a tensão de cisalhamento e a taxa de cisalhamento. A tensão de escoamento foi estimada pela extrapolação da curva de tensão versus taxa de cisalhamento até a taxa $0 \mathrm{~s}^{-1}$.

\section{Ensaio oscilatório}

O ensaio oscilatório é indicado quando se pretende avaliar a cinética da hidratação do cimento sem afetar a estrutura que se forma continuamente com o tempo, graças à capacidade de aplicação de pequenas deformações, mantém a estrutura em repouso. Esse tipo de ensaio tem sido utilizado para avaliar as propriedades das pastas de cimento desde a mistura até o início de pega do cimento (SCHULTZ, 1991; SCHULTZ; STRUBLE, 1993; NACHBAUR et al., 2001; SAAK et al., 2001; WINNEFELD; HOLZER, 2003; BETIOLI et al., 2008, 2009).

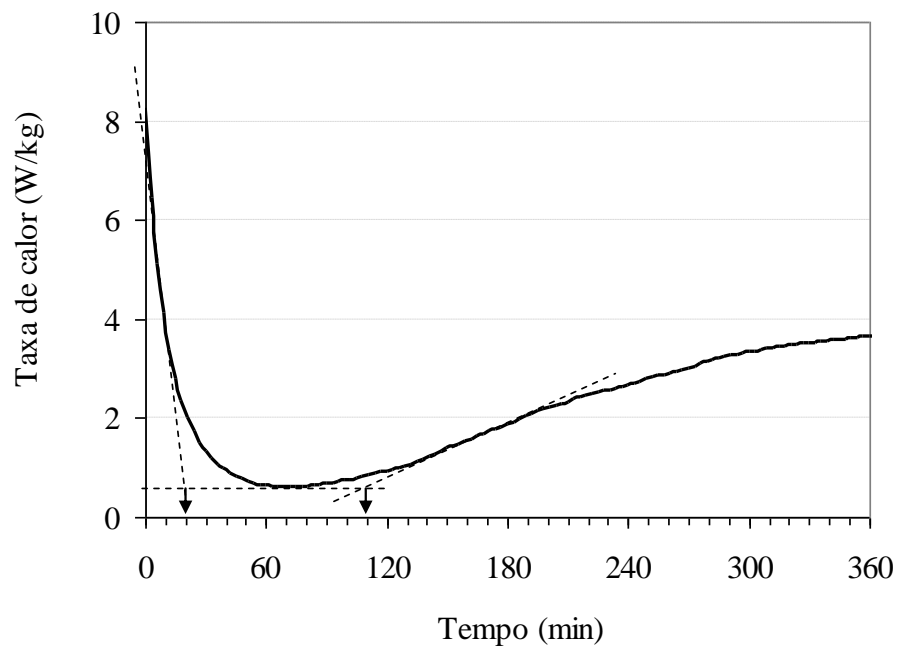

Figura 2 - Taxa de calor liberado durante 6 h de hidratação. 0 intervalo do período de indução ocorre entre 20 min e $110 \mathrm{~min}$, aproximadamente

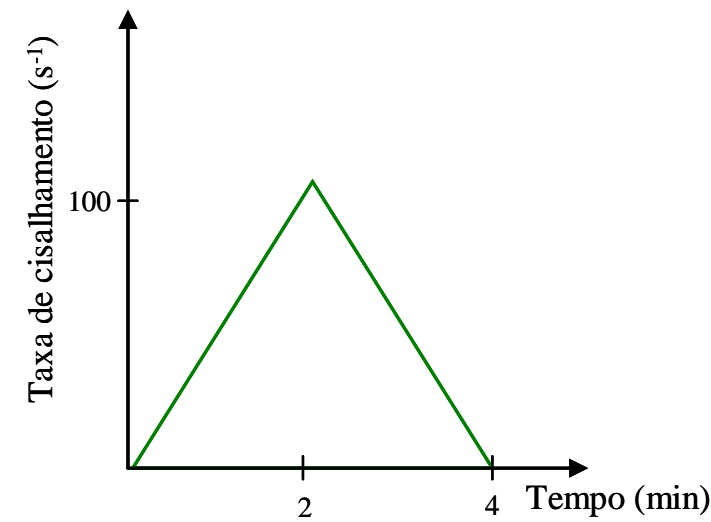

Figura 3 - Ensaio de fluxo, rampa de 0 a $100 \mathrm{~s}^{-1}$ em 2 min e até $0 \mathrm{~s}^{-1}$ em mais 2 min, realizado aos 15 min e 60 min de hidratação, utilizando-se uma amostra para cada tempo 
Neste ensaio o material é submetido a baixas deformações, menores que a deformação crítica (deformação máxima aplicada a um material sem perturbar a estrutura que se forma continuamente com o tempo), garantindo que as medidas sejam realizadas dentro do regime viscoelástico linear (RVL), no qual o módulo elástico independe da deformação aplicada. Os módulos elástico (ou módulo de armazenamento - G') e viscoso (ou módulo de perda - G”) podem ser determinados (BARNES et al., 1993).

Aplicando-se uma deformação senoidal sobre a amostra (Equação 1), obtém-se como resposta a essa solicitação a tensão (Equação 2) (BARNES et al., 1993).

$\gamma(t)=\gamma_{0} \times \cos (\omega t)$

$\tau=\tau_{0} \times \cos (\omega t+\delta)$

onde $\omega$ é a velocidade angular, t é o tempo, $\gamma_{\mathrm{o}}$ é a amplitude máxima de deformação e $\delta$ é o ângulo de fase.

Dividindo-se a tensão pela deformação, o resultado é um número complexo $\mathrm{G}^{*}\left(G^{*}=G^{\prime}+G^{\prime \prime}\right)$, o qual pode ser divido em duas componentes, real e imaginária. A componente real, elástica, é denominada de módulo de armazenamento (G'), e a componente imaginária, viscosa ou módulo de perda (G'”).

$G^{\prime}={ }_{0}^{\tau} /{ }_{\gamma 0} \cos \delta$

$G^{\prime \prime}={ }_{0}^{\tau} /{ }_{\gamma 0} \sin \delta$

Os comportamentos-limite para os materiais são sólido hookeano, quando a tensão está em fase com a deformação $(\delta=0)$, e fluido newtoniano, no qual a tensão está defasada em relação à deformação $\left(\delta=90^{\circ}\right)$. A maioria dos materiais existentes não apresenta comportamento de sólidos ou líquidos ideais, mas, sim, uma combinação dos mesmos, denominados de materiais viscoelásticos $\left(0<\delta<90^{\circ}\right)$.

A tensão de escoamento $\left(\tau_{0 \text { osc }}\right)$ pôde ser estimada pelo ensaio de varredura de deformação utilizandose a Equação 5, proposta por Schultz (1991), aos 15 min de hidratação.

$\tau_{\text {oosc }}=G^{\prime} \times \gamma_{\text {crit }}$

onde $G^{\prime}$ é o valor da componente elástica na deformação crítica $\left(\gamma_{\text {crít }}\right)$.

Essa propriedade foi estimada aos $60 \mathrm{~min}$, pela multiplicação da deformação crítica e o valor da componente elástica nesse intervalo de tempo.

Os ensaios oscilatórios foram realizados no mesmo equipamento que o ensaio de fluxo. $\mathrm{O}$ ensaio foi realizado em duas etapas: determinação da deformação crítica pelo ensaio de varredura de deformação, aumentando-se a amplitude de $10^{-5}$ a $10^{-1}$ sob frequência constante de $1 \mathrm{~Hz}$; ensaio de varredura de tempo, no qual a amplitude foi de $10^{-4}$ (menor que a deformação crítica) e frequência de 1 $\mathrm{Hz}$ por 60 min, conforme visualizado na Figura 4.

\section{Squeeze-flow}

O trabalho pioneiro utilizando o ensaio de squeezeflow em materiais cimentícios foi realizado por Mine et al. (1994) em pastas de cimento. Toutou, et al. (2005) utilizaram o método em argamassas de extrusão; Phan et al. (2006), em pasta de concreto autoadensável; Cardoso et al. (2005), Cardoso (2007) e Cardoso et al. (2009), em argamassas de revestimento; Betioli (2007), em pastas modificadas com polímeros usualmente utilizados em argamassas colantes.

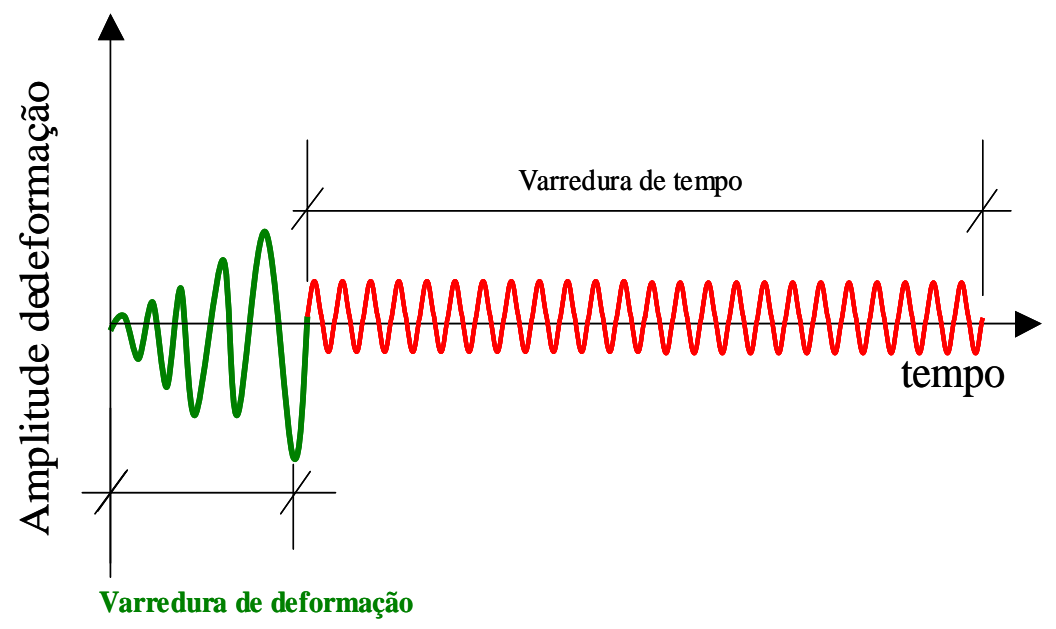

Figura 4 - Ensaio de varredura de deformação $\left(10^{-5}\right.$ a $\left.10^{-1}\right)$ e frequência de $1 \mathrm{~Hz}$, seguido de ensaio de varredura de tempo com deformação de $10^{-4}$ e frequência de $1 \mathrm{~Hz}$ até 60 minutos 
Essa técnica baseia-se na medida do esforço necessário para a compressão axial de uma amostra cilíndrica entre duas placas paralelas, por força ou deformação controlada. É um ensaio de fácil execução, que oferece repetibilidade (KOLENDA et al., 2003; CARDOSO et al., 2005; PHAN et al., 2006) e fornece resultados de carga em função do tempo e do deslocamento. $\mathrm{Na}$ prática, essa técnica simula as condições de fluxo sofrido pela pasta de cimento entre os agregados finos e graúdos no interior de argamassas e concretos, por exemplo.

O princípio fundamental deste método baseia-se no fato de que a deformação efetiva do material ocorre por cisalhamento radial quando a razão entre o diâmetro (D) e a espessura da amostra (h) for elevada $(\mathrm{D} / \mathrm{h}>5)$. Razões inferiores levam ao surgimento de tensões de compressão (MEETEN, 2004).

O perfil típico de uma curva carga versus deslocamento obtida no ensaio de squeeze-flow, com controle por deslocamento, pode apresentar três regiões bem definidas (Figura 5). A primeira região, sob pequenas deformações, é caracterizada por um comportamento elástico linear; a segunda, na qual o material pode se deformar consideravelmente com pequeno aumento na força aplicada, é caracterizada por deformação plástica ou fluxo viscoso; e a região três, na qual a força exigida para comprimir o material aumenta consideravelmente, é conhecida como o estágio de enrijecimento por deformação. A deformação que ocorre no segundo e no terceiro estágios depende de algumas características do sistema, tais como a distância e a fricção entre as partículas (MIN et al., 1994).

O ensaio de squeeze-flow pode gerar os parâmetros reológicos fundamentais, como viscosidade e tensão de escoamento. A viscosidade elongacional $\left(\eta_{\mathrm{B}}\right)$ é calculada conforme a Equação 6 (STEFFE, 1996).

$\eta_{B}=\sigma_{\mathrm{B}} /{ }_{\varepsilon}=2 L\left[h_{0}-{ }^{(v t)} /{ }_{v \pi R}{ }^{2}\right]$

onde $\sigma_{\mathrm{B}}$ é a tensão, ${ }_{\varepsilon}$ é a taxa de deformação elongacional, $\mathrm{L}$ é a carga, $\mathrm{h}_{0}$ é a altura inicial da amostra, $v$ é a velocidade do punção, t é o tempo e $\mathrm{R}$ é o raio do punção.

Para determinar a tensão de escoamento, primeiramente os dados de carga são transformados em tensão, dividindo-se a carga pela área do punção. A parte inicial da curva tensão versus deformação é ampliada para se determinar a transição entre o estágio I (elástico) e o estágio II (plástico) (HOPPE et al., 2006). O ponto exato da transição dos dois estágios pode ser relacionado com a tensão de escoamento do material, que pode então ser calculada por meio de um artifício geométrico. Esse procedimento consiste em se determinar a intersecção da extrapolação linear das curvas dos estágios I e II, como demonstrado nos resultados (Figura 11).

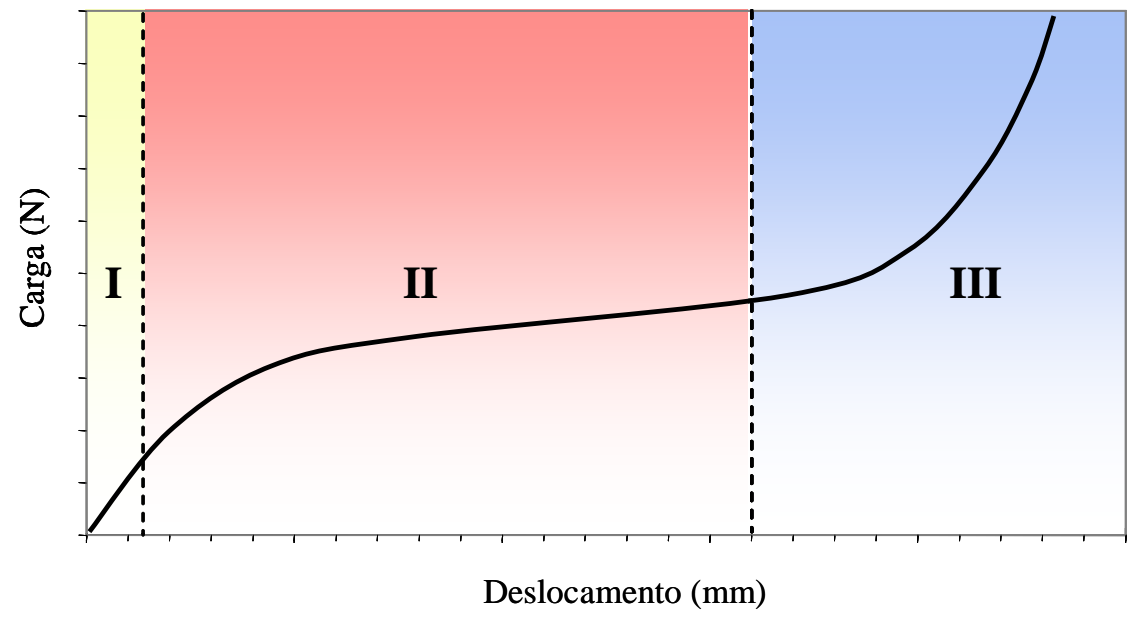

Nota: a região I equivale à deformação elástica; a região II, à deformação plástica ou fluxo viscoso; e a região III equivale ao enrijecimento por deformação

Figura 5 - Perfil típico de uma curva carga versus deslocamento de um ensaio de squeeze-flow com controle de deformação 


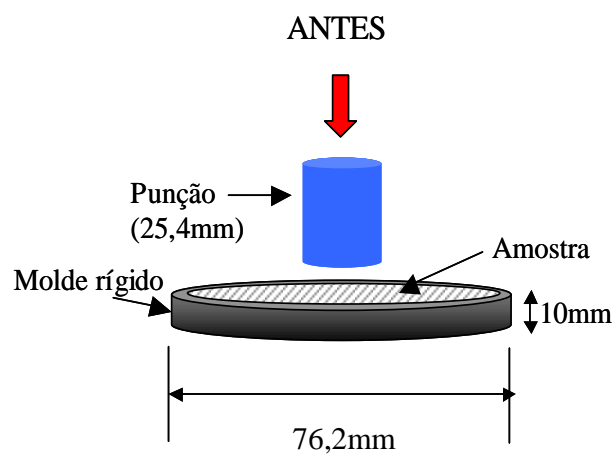

DEPOIS

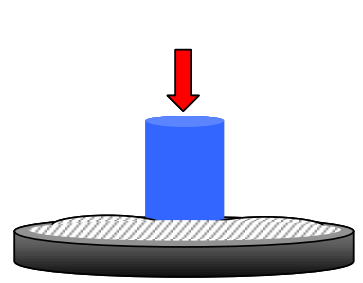

Nota: a pasta é comprimida até atingir $3,5 \mathrm{~mm}$ de penetração com velocidade de $0,05 \mathrm{~mm} / \mathrm{s}$

Figura 6 - Ilustração esquemática do ensaio de squeeze-flow

Como a pasta de cimento ainda no estado fresco não possui tensão de escoamento suficiente, os testes de squeeze-flow foram realizados em amostras confinadas, conforme utilizado por Min, Erwin e Jennings (1994), como mostra a Figura 6. Esses autores determinaram que, utilizando-se uma relação de diâmetro (Damostra/Dpunção ${ }^{1}$ ) maior que 1,7 , as forças de repulsão causadas pelo efeito parede durante o esmagamento podem ser negligenciadas.

Neste trabalho utilizou-se uma amostra com diâmetro de 76,2 $\mathrm{mm}$ e altura de $10 \mathrm{~mm}$ (molde feito com anel de PVC) e um punção de diâmetro de 25,4 mm, conforme a Figura 6 (relação entre os diâmetros igual a 3 e relação entre diâmetro da amostra e altura > 5). Tanto o punção quanto o prato de apoio da amostra são de aço inoxidável.

$\mathrm{O}$ ensaio foi realizado em uma máquina universal de ensaios Instron (modelo 5569), com deslocamento controlado, utilizando-se células de carga de $10 \mathrm{~N}$ e $1.000 \mathrm{~N}$. A deformação de $3,5 \mathrm{~mm}$ foi aplicada com velocidade constante de 0,05 $\mathrm{mm} / \mathrm{s}$. Os dados de carga, deslocamento e tempo foram coletados e registrados pelo software do equipamento numa taxa de 10 pontos por segundo. Todos os ensaios foram realizados segundo o conceito de compressão simples, controlada por deslocamento.

\section{Resultados e discussões}

\section{Ensaio de fluxo}

A Figura 7 mostra as curvas de tensão versus taxa de cisalhamento (a) e viscosidade aparente versus taxa de cisalhamento (b) para as pastas de cimento aos 15 min e 60 min de hidratação.

O perfil da curva tensão versus taxa de cisalhamento aos 15 min é típico de materiais com estrutura aglomerada (Figura 7a). Com o aumento da taxa de cisalhamento, a estrutura tridimensional de partículas de cimento se rompe (BARNES et al., 1993; NONAT et al., 1997; SAAK et al., 2001), provavelmente devido à redução do tamanho das unidades móveis e, por isso, a tensão de cisalhamento diminui sob altas taxas.

Observa-se ainda que todas as pastas apresentam certo grau de pseudoplasticidade, em que a viscosidade aparente, calculada pela relação entre a tensão e taxa de cisalhamento, diminui com o aumento da taxa de cisalhamento (Figura 7b). Após $60 \mathrm{~min}$, o mesmo comportamento é observado, porém com maiores valores de tensão de cisalhamento, indicando a presença de uma estrutura mais aglomerada, resultado dos efeitos de consolidação, isto é, atuação de fenômenos físicos (fenômenos de aglomeração) e, como menor intensidade, de fenômenos químicos (reações de hidratação), pois as medidas foram realizadas durante o período de indução (Figura 2).

A tensão de escoamento, propriedade diretamente ligada ao estado de aglomeração das partículas (SAAK et al., 2001), dobrou de valor entre os tempos 15 min e 60 min de hidratação (Tabela 4), resultado dos efeitos de consolidação (BETIOLI $e t$ al., 2008).

A taxa de cisalhamento promove a ruptura da estrutura de partículas que se forma. Dessa forma, o ensaio de fluxo não é apropriado para avaliar as características reológicas de pastas cimentícias quando o objetivo é avaliar a consolidação em função do tempo. Nesse caso, ensaios dinâmicos como o ensaio oscilatório, são os mais adequados.

\footnotetext{
${ }^{1}$ Aplicador de carga no material.
} 


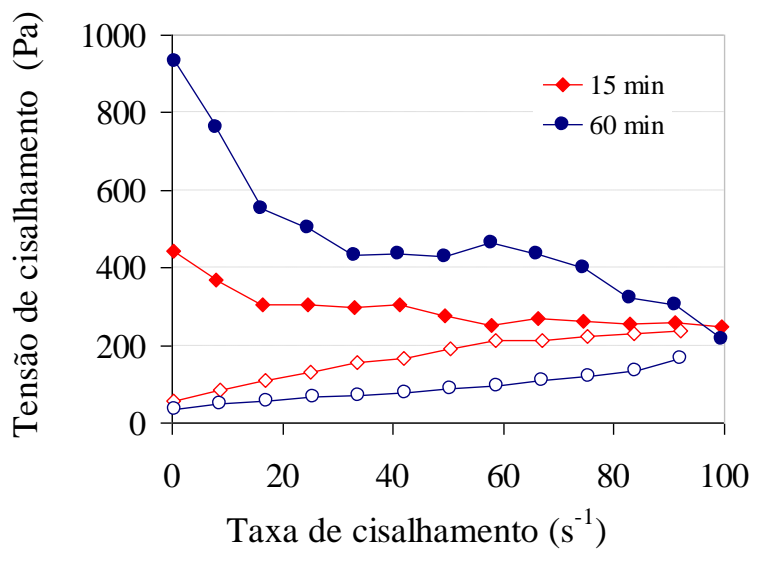

(a)

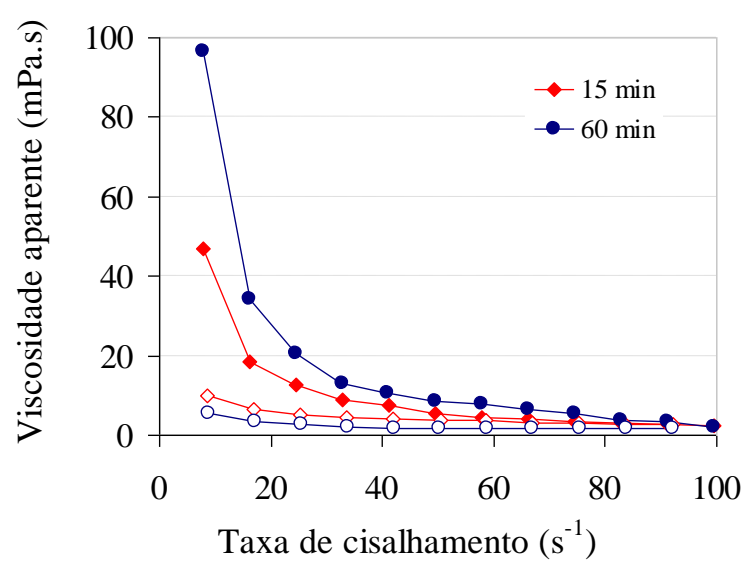

(b)

Nota: os símbolos cheios indicam o aumento da taxa de cisalhamento, e os vazios, a redução da taxa de cisalhamento

Figura 7 - Ciclos de histerese: tensão versus taxa de cisalhamento da pasta de cimento (a) e valores de viscosidade aparente em função da taxa de cisalhamento e da pasta de cimento aos 15 min e 60 min (b)

\begin{tabular}{c|c|c|c|c|c}
\hline \multicolumn{2}{c|}{$\tau_{\mathbf{0} \text { fluxo }}(\mathbf{k P a})$} & \multicolumn{2}{c|}{$\tau_{\mathbf{0} \text { oscilatótio }}(\mathbf{k P a})$} & \multicolumn{2}{c}{$\sigma_{\mathbf{0} \text { Squeeze-flow }}(\mathbf{k P a})$} \\
\hline $15 \mathrm{~min}$ & $60 \mathrm{~min}$ & $15 \mathrm{~min}$ & $60 \mathrm{~min}$ & $15 \mathrm{~min}$ & $60 \mathrm{~min}$ \\
\hline 0,45 & 0,90 & 0,043 & 0,10 & 0,10 & 0,50 \\
\hline
\end{tabular}

Tabela 4 - Valores de tensão de escoamento da pasta de cimento $(a / c=0,38)$ determinados por diferentes ensaios reológicos

\section{Ensaio oscilatório}

A pasta de cimento exibiu uma região viscoelástica linear (RVL) com pequena deformação crítica, de aproximadamente $3,7 \times 10^{-4}$ aos $15 \mathrm{~min} \mathrm{de}$ hidratação, determinada pelo ensaio de varredura de deformação. Essa baixa deformação é usualmente relacionada com suspensões aglomeradas, em que forças de curto alcance atuam formando uma rede tridimensional de partículas em meio aquoso. Essas forças não suportam deformações maiores que a deformação crítica sem que haja rompimento (SCHULTZ; STRUBLE, 1993; NACHBAUR et al., 2001).

O comportamento viscoelástico da pasta de cimento foi determinado pelo ensaio de varredura de tempo (deformação constante de $10^{-4}$, menor que a deformação crítica e frequência de $1 \mathrm{~Hz}$ ). A Figura 8 mostra a evolução da componente elástica $\left(G^{\prime}\right)$ e da componente viscosa (G') da pasta em função do tempo. $\mathrm{O}$ valor da componente elástica $\left(\mathrm{G}^{\prime}\right)$ foi sempre maior que o da componente viscosa (G'), o que significa que o comportamento sólido é predominante já nos primeiros 20 min de hidratação.

Esses resultados confirmam a intensa consolidação promovida pelas forças atrativas eletrostáticas e de van der Waals entre as partículas de cimento
(YOSHIOKA et al., 2002), as quais aumentam com o aumento da força iônica, em função da dissolução do cimento (NACHBAUR et al., 1998), e com a formação dos produtos hidratados (TAYLOR, 1990; THOMAS et al., 1998). Esse último fator é pouco significante pelo fato de a medida ter sido realizada no período de indução.

Observa-se na Tabela 4 que a tensão de escoamento por esse método foi 10 vezes menor que a estimada pelo ensaio de fluxo. Tal diferença prova a menor condição de cisalhamento do método oscilatório durante as medidas reológicas.

A associação dos ensaios de fluxo e oscilatório melhora a compreensão dos fenômenos envolvidos no comportamento de suspensões cimentícias sob fluxo e durante sua consolidação. Entretanto, ambos os métodos não avaliam as mudanças geométricas a que as pastas de cimento podem estar sujeitadas quando comprimidas entre agregados e durante diferentes processos de aplicação, como extrusão e deformação por compressão. Dessa forma, a pasta de cimento foi avaliada igualmente por ensaio squeeze-flow, um método convencional para a caracterização reológica de alimentos e compósitos (STEFFE, 1996; MEETEN, 2000), mas que vem ganhando campo de aplicação em estudos de argamassa e pastas de cimento, conforme já comentado. 


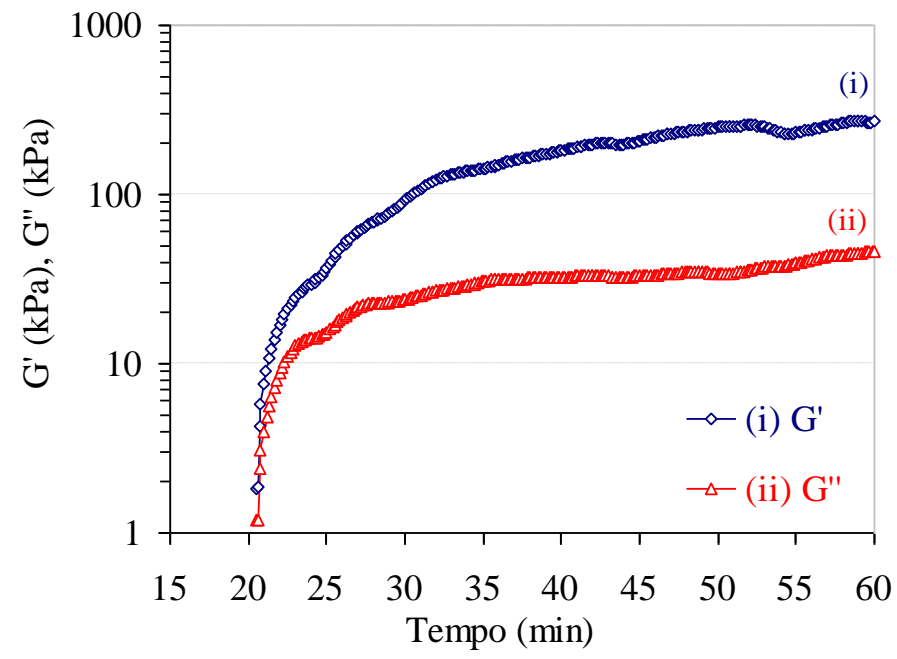

Figura 8 - Componentes elástica (G') e viscosa (G”) da pasta de cimento em função do tempo de hidratação após ensaio de varredura de deformação

\section{Squeeze-flow}

A Figura 9 mostra o perfil das pastas de cimento aos 15 min e 60 min de hidratação. Aos 15 min a carga aumenta linearmente até $1,5 \mathrm{~mm}$ de deformação (estágio I) e acima dessa deformação observa-se um enrijecimento por deformação, em que a força requerida para comprimir o material aumenta rapidamente (estágio III), atingindo o valor de $2,2 \mathrm{~N}$ a $3,5 \mathrm{~mm}$.

O enrijecimento se deve ao fato de que, como a distância entre as placas se reduz durante o ensaio, o confinamento das partículas na direção vertical reduz a distância de separação entre elas; consequentemente, as forças de atrito tornam-se mais intensas.

Depois de 60 min, o enrijecimento por deformação foi mais evidente e apresentou um perfil exponencial, iniciando em deformações mais baixas $(0,75 \mathrm{~mm})$, alcançando $21 \mathrm{~N}$ aos $3,5 \mathrm{~mm}$ (não mostrado no gráfico), um aumento de quase 10 vezes.

Os resultados de squeeze-flow analisados em termos de carga e deslocamento são suficientes para avaliar o comportamento de diferentes materiais. No entanto, essa técnica permitiu ainda a determinação dos parâmetros reológicos como a viscosidade elongacional (Figura 10) e a tensão de escoamento (Tabela 4).

O aumento de viscosidade com a taxa de cisalhamento não é um comportamento dilatante (quando a viscosidade aparente aumenta com o aumento da taxa ou da tensão de cisalhamento). Como os testes foram realizados com taxa de deformação constante, a variação na taxa de deformação elongacional é causada pela redução da altura da amostra. Então, a cada nova altura, uma nova solicitação ocorre continuamente na amostra, uma vez que a distribuição das partículas e do fluido modifica-se constantemente para promover o fluxo do material como um todo. Essa diferença particular faz com que o método tenha capacidade de identificar a influência de características relacionadas com o atrito no comportamento reológico de suspensões concentradas.

A tensão de escoamento também foi determinada neste ensaio por meio de um artifício geométrico. Esse procedimento consiste em se determinar a intersecção da extrapolação linear das curvas dos estágios I e II, conforme ilustrado na Figura 11 para a pasta de cimento pura aos $15 \mathrm{~min}$ de hidratação. 


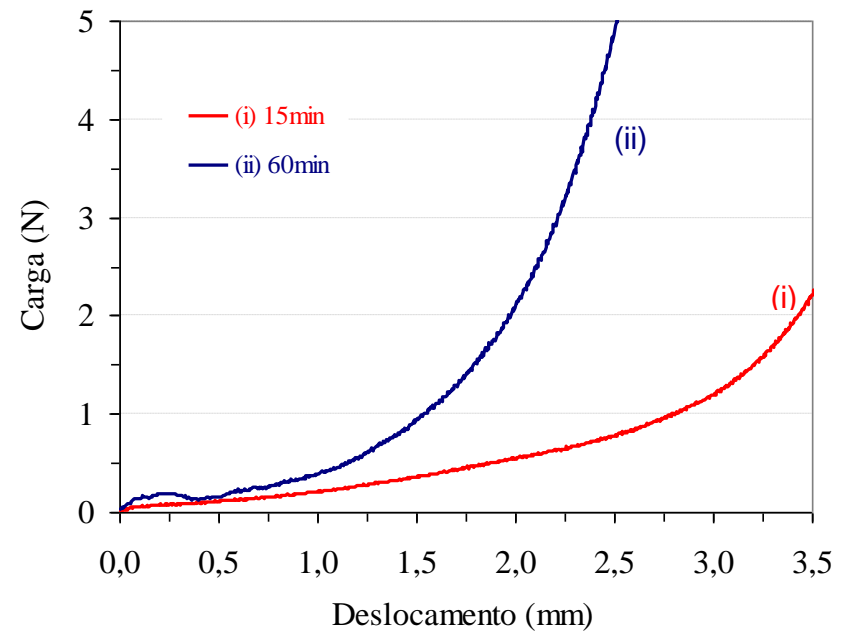

Figura 9 - Carga versus deslocamento - resultados do ensaio de squeeze-flow na pasta de cimento aos 15 min e 60 min de hidratação

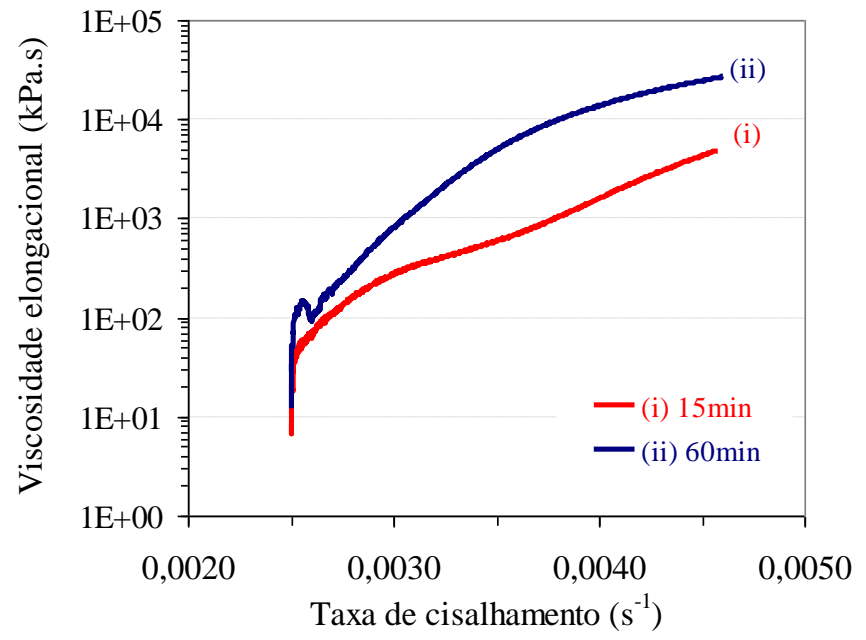

Figura 10 - Viscosidade elongacional versus taxa de cisalhamento elongacional para a pasta de cimento aos 15 min e 60 min de hidratação

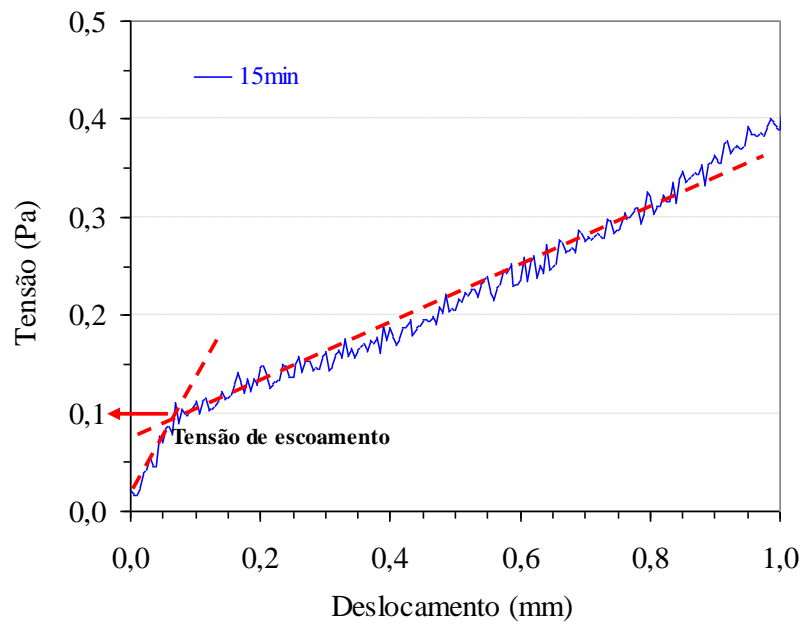

Figura 11 - Procedimento geométrico adotado para determinar a tensão de escoamento no ensaio de squeeze-flow 
A pasta de cimento apresentou uma tensão de escoamento de $0,10 \mathrm{kPa}$ e de $0,50 \mathrm{kPa}$ aos $15 \mathrm{~min}$ e $60 \mathrm{~min}$, respectivamente. Como esperado, a consolidação da pasta aumentou a tensão de escoamento. Conforme a Tabela 4 , os valores de tensão de escoamento foram menores que os do ensaio de fluxo, mas superiores aos valores do ensaio oscilatório. Isso significa que o squeezeflow tem uma menor condição de cisalhamento que o ensaio de fluxo; no entanto, maior que o oscilatório.

\section{Conclusões}

O comportamento reológico de materiais à base de cimento é complexo devido aos diferentes tipos (concretos, argamassas, fibrocimento), condições de misturas (velocidade, tempo, confinamento) e aplicações (extrusão, autoadensável, bombeamento, etc.), além da natureza reativa do cimento. Essa variedade de materiais/aplicações requer diferentes características reológicas.

Cada método reológico utilizado neste trabalho forneceu uma solicitação com diferente magnitude de cisalhamento: alta para ensaio de fluxo (simular processos de mistura e aplicação) e squeeze-flow (simular formação por extrusão, por compressão); e baixa para oscilatório (avaliar fenômenos relacionados à pega e consolidação). Isso resultou em diferentes valores das propriedades reológicas (viscosidade e tensão de escoamento).

A tensão de escoamento foi maior no ensaio de fluxo, seguido do ensaio de squeeze-flow e oscilatório, comprovando o menor cisalhamento do material neste último ensaio. Já com relação à viscosidade, sua comparação não pode ser direta, uma vez que no squeeze-flow a mudança na taxa de deformação é causada pela redução da distância entre as placas; e no ensaio de fluxo, a medida é função direta da taxa de cisalhamento aplicada. Porém, observou-se uma diferença de ordens de grandeza.

Este trabalho mostrou que a caracterização reológica de uma pasta de cimento requer uma associação de técnicas complementares, abordagem pouco explorada na literatura, que permita identificar o comportamento de materiais cimentícios sob diferentes solicitações.

\section{Referências}

BARNES, H. A.; HUTTON, J. F.; WALTERS, K. An Introduction to Rheology. 3. ed. Amsterdam: Elsevier, 1993. 199 p.
BETIOLI, A. M. Influência dos Polímeros

MHEC e EVA na Hidratação e

Comportamento Reológico de Pastas de

Cimento Portland. 2007. 188 f. Tese (Doutorado

em Engenharia Civil) - Departamento de

Engenharia Civil, Universidade Federal de Santa

Catarina, Florianópolis, 2007.

BETIOLI, A. M. et al. Relação entre o

Comportamento Reológico e os Fenômenos

Físico-Químicos no Início da Hidratação do

Cimento Portland com adição de Filler. In:

ENCONTRO NACIONAL DE TECNOLOGIA

DO AMBIENTE CONSTRUÍDO, 12., 2008,

Fortaleza. Anais... Fortaleza: ENTAC, 2008.

BETIOLI, A. M. et al. Effect of HMEC on the Consolidation of Cement Pastes: isothermal calorimetry versus oscillatory rheometry. Cement and Concrete Research, v. 39, n. 5, p. 440-445, maio 2009.

CARDOSO, F. A.; PILEGGI, R. G.; JOHN, V. M. Caracterização Reológica de Argamassas pelo Método de Squeeze-Flow. In: SIMPÓSIO

BRASILEIRO DE TECNOLOGIA DE ARGAMASSAS, 1.; INTERNATIONAL SYMPOSIUM ON MORTARS TECHNOLOGY, 6., 2005, Florianópolis. Anais... Florianópolis: ANTAC, 2005. p. 121-143.

CARDOSO, F. A. et al. Caracterização Reológica de Argamassas do Mercado por Squeeze-Flow. In: SIMPÓSIO BRASILEIRO DE TECNOLOGIA DE ARGAMASSAS, 7., Recife, 2007. Anais... Recife: ANTAC, 2007.

CARDOSO, F. A.; JOHN, V. M.; PILEGGI, R. G. Rheological Behavior of Mortars under Different Squeezing Rates. Cement and Concrete

Research, v. 39, n. 9, p. 748-753, set. 2009.

ENGMANN, J.; SERVAIS, C.; BURBIDGE, A. S. Squeeze Flow Theory and Applications to Rheometry: a review. Journal Non-Newtonian Fluid Mechanical, v. 132, n. 1/3, p. 149-175, dez. 2005.

HOPPE F, J. et al. Técnicas de Caracterização Reológica de Argamassas. E-Mat (Porto Alegre), v. 3, p. 103-120, 2006.

JIANG, S. P.; MUTIN, J. C.; NONAT, A. Studies on Mechanism and Physico-Chemical Parameters at the Origin of the Cement Setting: the fundamental processes involved during the cement setting. Cement and Concrete Research, v. 25, n. 4, p. 779-789, maio 1995. 
KOLENDA, F. et al. Identification of Rheological Parameters by the Squeezing Test. Powder Technology, v. 130, n. 1, p. 56- 62, fev. 2003.

MEETEN, G. H. Yield Stress of Structured Fluids Measured by Squeeze Flow. Rheologica Acta, Berlim, v. 39, n. 4, p. 399-408, 2000.

MEETEN, G. H. Squeeze Flow of Soft Solids Between Rough Surfaces. Rheologica Acta, Berlim, v. 43, n. 1, p. 6 - 16, fev. 2004.

MIN, B. H., ERWIN, L. JENNINGS, H. M. Rheological Behavior of Fresh Cement Paste as Measured by Squeeze Flow. Journal of Materials Science, v. 29, n. 5, p. 1374-1381, mar. 1994.

NACHBAUR, L. et al. Electrokinetic Properties which Control the Coagulation of Silicate Cement Suspensions During Early Ages Hydration. Journal of Colloid and Interface Science, v. 202, n. 2, p. 261-268, jun. 1998.

NACHBAUR, L. et al. Dynamic Mode Rheology of Cement and Tricalcium Silicate Pastes from Mixing to Setting. Cement and Concrete Research, v. 31, n. 2, p 183-192, fev. 2001.

NONAT, A. et al. Physico-Chemical Parameters Determining Hydration and Particle Interactions During the Setting of Silicate Cements. Solid State Ionics, v. 101-103, n. 2, p. 923-930, nov. 1997.

ÖZKAN, N. et al. Rheological Analysis of Ceramic Pastes. Journal of European Ceramic Society, v. 19, n. 16, p. 2883-2891, 1999.

PHAN, T. H.; CHAOUCHE, M.; MORANVILLE, M. Squeeze Flow of Self-Compacting Cement Pastes. In: INTERNATIONAL SYMPOSIUM ON ADVANCES IN CONCRETE THROUGH SCIENCE AND ENGINEERING, 2., 2006, Quebec. Anais... Quebec: RILEM, 2006.

SAAK, A. W.; JENNINGS, H. M.; SHAH, S. P. The Influence of Wall Slip on Yield Stress and Viscoelastic Measurements of Cement Paste. Cement and Concrete Research, v. 31, n. 2, p. 205-212, fev. 2001.

SCHULTZ, M. A. Rheological Studies of Fresh Cement Pastes. Illinois, 1991. 192 f. Dissertação (Mestrado em Engenharia Cerâmica) - University of Illinois at Urbana-Champaign, 1991.

SCHULTZ, M. A.; STRUBLE, L. J. Use of Oscillatory Shear to Study Flow Behavior of Fresh Cement Paste. Cement and Concrete Research, v. 23, n. 2, p. 273-282, 1993.
SILVA, D. A. Efeitos dos Polímeros HEC e EVA na Microestrutura de Pastas de Cimento Portland. 2001. Tese (Doutorado em Ciência e Engenharia dos Materiais) - Departamento de Engenharia dos Materiais, Universidade Federal de Santa Catarina, Florianópolis, 2001.

STEFFE, J. F. Rheological Methods in Food Process Engineering. 2nd. East Lansing: Freeman Press, 1996. 418p.

TAYLOR, H. F. W. Cement Chemistry. Londres: Academic Press Limited, 1990.

THOMAS, J. J.; JENNINGS, H. M.; ALLEN, A. J. The Surface Area of Cement Paste as Measured by Neutron Scattering: evidence for two C-S-H morphologies. Cement and Concrete Research, v. 28, n. 6, p. 897-905, 1998.

TOUTOU, Z.; ROUSSEL, N.; LANOS, C. The Squeezing Test: a tool to identify firm cementbased material's rheological behavior and evaluate their extrusion ability. Cement and Concrete Research, v. 35, n. 10, p. 1891-1899, 2005.

WINNEFELD, F.; HOLZER, L. Monitoring Early Cement Hydration by Rheological Measurements. In: INTERNATIONAL CONGRESS ON THE CHEMISTRY OF CEMENT, 11., 2003, Durban, Africa do Sul. Anais... Durban: ICCC, 2003.

\section{YANG, M.; JENNINGS, H. M. On the}

Development of Rheological Properties of Cement Paste During the Induction Period In:

MATERIALS RESEARCH SOCIETY

SYMPOSIUM PROCEEDINGS-FLOW AND

MICROSTRUCTURE OF DENSE

SUSPENSIONS, 1992, Boston. Anais... Boston: MRS, 1992.

YOSHIOKA K. et al. Adsorption Characteristics of Superplasticizers on Cement Component Minerals. Cement and Concrete Research, v. 32, n. 10, p. 1507-1513, 2002.

\section{Agradecimentos}

Os autores agradecem à CAPES pelo aporte financeiro, à DpUnion pelo empréstimo do reômetro AR2000 (TA Instrument) e ao Laboratório de Microestrutura (LME) do Departamento de Engenharia de Construção Civil e Urbana da EPUSP por disponibilizar a infraestrutura necessária para realizar esta pesquisa. 